\title{
Association of HDL-C and apolipoprotein A-I with the risk of type 2 diabetes in subjects with impaired fasting glucose
}

\author{
You-Cheol Hwang, Hong-Yup Ahn', Sung-Woo Park² and Cheol-Young Park ${ }^{2}$ \\ Division of Endocrinology and Metabolism, Department of Medicine, Kyung Hee University School of Medicine, \\ Kyung Hee University Hospital at Gangdong, Seoul, Republic of Korea, ${ }^{1}$ Department of Statistics, Dongguk \\ University-Seoul, Seoul, Republic of Korea and ${ }^{2}$ Division of Endocrinology and Metabolism, Department of Internal \\ Medicine, Sungkyunkwan University School of Medicine, Kangbuk Samsung Hospital, Pyung-Dong, Jongro-Gu, \\ 110-746 Seoul, Republic of Korea
}

Correspondence should be addressed to C-Y Park

Email

cydoctor@chol.com

\begin{abstract}
Objectives: HDLs have many diverse functions. The goal of this study was to determine the association of HDL cholesterol (HDL-C) and apolipoprotein A-I (apoA-I) with the development of type 2 diabetes (T2D). In particular, this study determined the association between the ratio of HDL-C to apoA-I (HA) and incident T2D.

Design and methods: A total of 27988 subjects with impaired fasting glucose (IFG) (18 266 men and 9722 women) aged 21-91 years (mean age 40.7 years) were followed for a mean duration of 2.81 years.

Results: Study subjects were divided into quartiles according to the baseline HA ratio. Age, male sex, current smoking, BMI, waist circumference, and high-sensitivity C-reactive protein decreased across the quartiles, and all metabolic profiles, including blood pressure, fasting glucose, insulin resistance as determined by homeostasis model assessment of insulin resistance, and lipid measurements such as total cholesterol, LDL cholesterol, non-HDL-C, and apoB, improved as the HA ratio increased. In addition, incident cases of T2D decreased as the HA ratio increased, independent of age, sex, BMI, current smoking, systolic blood pressure, $\mathrm{HbA1c}$, fasting serum insulin, family history of diabetes, and serum triglyceride concentrations (HR $(95 \% \mathrm{Cl})$ of fourth quartile vs first quartile; $0.76(0.67-0.86), P<0.0001)$. Conclusions: A higher HA ratio was associated with favorable metabolic profiles and a lower risk of T2D development in subjects with IFG.
\end{abstract}

\section{Introduction}

Large epidemiological studies have demonstrated an inverse relationship between serum HDL cholesterol (HDL-C) concentrations and the risk of coronary heart disease (CHD) $(1,2)$, that is, each $1 \mathrm{mg} / \mathrm{dl}$ increase in HDL-C is associated with $\sim 2-3 \%$ lower risk of CHD (3). The primary mechanism for this inverse relationship is the ability of HDLs and apolipoprotein (apo) A-I to mediate reverse cholesterol transport from non-hepatic cells to the liver and steroidogenic organs (4). ApoA-I is the structural apolipoprotein in HDLs and constitutes about $70 \%$ of the apolipoprotein content of HDL particles (4); thus, plasma
apoA-I concentrations closely correlate with plasma HDL-C concentrations (5). However, the degree of this correlation varies and only $43 \%$ of the variance in HDL-C can be explained by variation in apoA-I concentrations (5).

It is well known that HDLs protect against atherosclerosis, but additional functions have also been reported, including anti-inflammatory, antioxidative, anti-apoptotic, vasodilatory, antithrombotic, and anti-infectious properties and direct modulation of glucose metabolism (6). Accordingly, recent epidemiological studies have demonstrated the association between low HDL-C concentrations
(ㄷ) 2014 European Society of Endocrinology Printed in Great Britain
Published by Bioscientifica Ltd. 
and the risk of incident type 2 diabetes (T2D) $(7,8)$. By contrast, while little is known about the role of apoA-I in the development of T2D, the findings of some recent studies have suggested that high serum apoA-I is associated with an increased risk of incident diabetes $(9,10)$.

Therefore, the goal of this study was to determine the role of HDL-C and apoA-I in the development of T2D in patients with impaired fasting glucose (IFG). Study subjects were divided into quartiles according to the baseline HDL-C to apoA-I (HA) ratio, and independent associations with the development of T2D were analyzed across the quartiles.

\section{Subjects and methods}

\section{Study population}

Study subjects were recruited from a population of patients who visited the Health Screening Center at Kangbuk Samsung Hospital between January 2005 and December 2010 for routine medical examinations. During this period, a total of 130409 subjects received medical examinations on at least two occasions. Among these patients, 6794 were excluded as they had T2D at baseline. In addition, seven subjects were excluded because anthropometric or lipid data were not available for them. Finally, a total of 123608 subjects were reviewed and a total of 27988 subjects with IFG (18 266 men and 9722 women) aged 21-91 years (mean age 40.7 years) were enrolled in this study. Subjects were followed for a mean of 2.81 years, with a range of 1-72 months.

The study protocol and data analysis were approved by the Institutional Review Board of Kangbuk Samsung Hospital. As the data did not include any personal information, the Board determined that the study was exempt from the need to obtain informed consent from study participants.

\section{Clinical and laboratory examination}

A complete physical examination was performed in which information about personal medical history, family history of diabetes, and lifestyle factors including cigarette smoking and alcohol consumption were obtained using a standardized questionnaire. Blood pressure was measured by trained nurses using a mercury sphygmomanometer on the right arm after a 5-min rest interval with the patient in a seated position. Weight and height were measured in the morning with subjects wearing light clothing and no shoes, and BMI was calculated as weight in kilograms divided by the square of the height in meters. Waist circumference was measured at a level midline between the lower rib margin and iliac crest.

All blood samples were obtained in the morning following an overnight fast of 12-14 h. Serum glucose was measured by the hexokinase method using an autoanalyzer (Advia 1800; Siemens, Berlin, Germany). The interassay coefficient of variation $(\mathrm{CV})$ value was $0.6-1.6 \%$. Serum insulin was measured using an IRMA (Biosource, Nivelles, Belgium), with intra- and inter-assay CV values of 2.1-4.5 and 4.7-12.2\% respectively. The homeostasis model assessment (HOMA), which is based on fasting glucose and insulin concentrations, was used to estimate insulin sensitivity and $\beta$-cell function (11). Serum total cholesterol, triglycerides, HDL-C, and directly measured LDL cholesterol levels were determined using an autoanalyzer (Advia 1800; Siemens). Serum apoB and apoA-I concentrations were determined by immunoturbidometric methods (Advia 2400, autoanalyzer; Siemens), with inter-assay $\mathrm{CV}$ values of $2.1-6.1$ and $1.8-4.8 \%$ respectively. High-sensitivity C-reactive protein (hs-CRP) was analyzed by particle-enhanced immunonephelometry with a BNIITM System (Dade Behring, Marburg, Germany). HbA1c levels were determined by immunoassays (Cobas Integra 800; Roche), with an inter-assay CV value of $0.9-3.3 \%$. The clinical laboratory used in this study participates in annual inspections and surveys by the Korean Association of Quality Assurance for Clinical Laboratories and has been accredited for quality control and performance of these measurements.

In this study, IFG was defined by the presence of one of the following: i) fasting glucose levels of $100-125 \mathrm{mg} / \mathrm{dl}$ or ii) HbA1c levels of 5.7-6.4\% (39-46 mmol/mol). Incident T2D was defined by the presence of one of the following: i) fasting glucose levels $\geq 126 \mathrm{mg} / \mathrm{dl}$; ii) treatment involving oral hypoglycemic agents or insulin therapy; iii) a self-reported history of diabetes; or iv) HbA1c levels $\geq 6.5 \%$ (48 mmol/mol) (12). Subjects with incident type 1 diabetes that had a history of ketoacidosis at initial presentation, who essentially required insulin therapy for blood glucose control, or who had low to undetectable serum insulin levels were excluded (12).

\section{Statistical analyses}

Data are expressed as mean \pm s.D. or as proportions. Differences among groups were tested by ANOVA for continuous variables and the $\chi^{2}$-test was used for categorical variables. A Kaplan-Meier survival curve was used to determine differences among groups in terms of 
the development of T2D. Multivariate Cox hazard regression analyses were performed to determine whether the HA ratio was associated with T2D independent of age, sex, BMI, current smoking, systolic blood pressure, HbA1c, fasting serum insulin, family history of diabetes, and serum triglyceride concentration. Analyses were performed using $R$ version 2.14.2 (http://www.r-project.org). $P$ values of $<0.05$ were considered statistically significant.

\section{Results}

The clinical characteristics of the study population according to the HA ratio quartile are given in Table 1 . Age, male sex, current smoking, BMI, waist circumference, and hs-CRP decreased across the quartiles, and all metabolic profiles, including blood pressure, fasting glucose, insulin resistance as determined by HOMA-IR, and lipid measurements such as total cholesterol, LDL-C, non-HDL-C, and apoB, improved as the HA ratio increased.

Serum HDL-C levels were independently associated with a lower incidence of T2D after adjusting for age and sex (HR per 1-s.D., 0.91; 95\% CI, 0.88-0.95; $P<0.0001$, Table 2). However, the protective effect of HDL-C disappeared after further adjusting for BMI, current smoking, systolic blood pressure, HbA1c, fasting serum insulin, family history of diabetes, and triglycerides. However, serum apoA-I levels were independently associated with a higher incidence of T2D even after adjusting for all of the aforementioned confounding factors (HR per 1-S.D., 1.10; 95\% CI, 1.07-1.15; $P<0.0001$, Table 2).

During follow-up, 4589 cases (16.4\%) of incident T2D developed in patients with IFG. Incident cases of T2D decreased as the HA ratio increased $(20.9 \%(n=1265)$, $18.2 \%(n=1101), 16.0 \%(n=965)$, and $14.9 \%(n=902)$ in the first, second, third, and fourth quartiles respectively; $P<0.001$, Fig. 1). This was independent of aforementioned risk factors for T2D (Table 3). In the final model (model V), subjects in the fourth quartile showed a $21 \%$ risk reduction compared with subjects in the first quartile for development of T2D (HR (95\% CI) of fourth quartile vs first quartile; 0.76 (0.67-0.86), $P<0.0001)$.

\section{Discussion}

Apart from the role of HDLs in reverse cholesterol transport, recent studies have uncovered a variety of

Table 1 Baseline characteristics of study subjects according to quartiles based on the ratio of HDL cholesterol to apolipoprotein A-I. Data are expressed as mean \pm s.D. or frequency (\%). Smoking data were available for 16573 subjects.

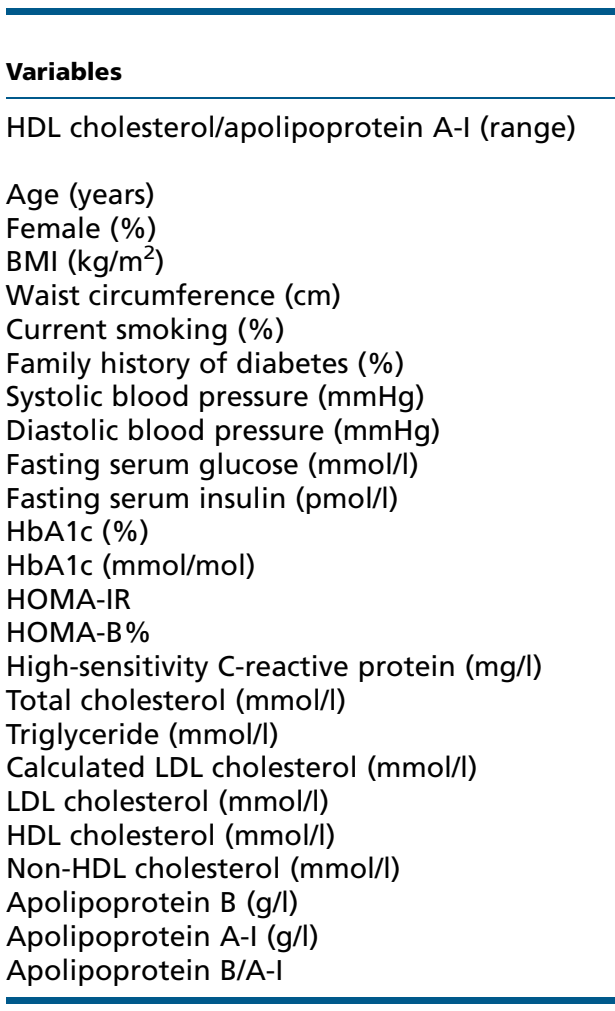

\begin{tabular}{c} 
First quartile \\
$(n=6997)$ \\
\hline $0.31 \pm 0.02$ \\
$(0.16-0.33)$ \\
$41.2 \pm 8.1$ \\
$1193(17.1)$ \\
$25.6 \pm 2.9$ \\
$86.6 \pm 8.1$ \\
$1561(37.7)$ \\
$797(11.4)$ \\
$119.5 \pm 14.1$ \\
$78.3 \pm 9.8$ \\
$5.7 \pm 0.4$ \\
$76.4 \pm 27.1$ \\
$5.6 \pm 0.3$ \\
$38 \pm 4$ \\
$2.80 \pm 1.05$ \\
$102.2 \pm 41.1$ \\
$0.14 \pm 0.28$ \\
$5.00 \pm 0.85$ \\
$2.33 \pm 1.39$ \\
$2.81 \pm 0.79$ \\
$2.85 \pm 0.70$ \\
$1.12 \pm 0.18$ \\
$3.88 \pm 0.82$ \\
$1.05 \pm 0.22$ \\
$1.41 \pm 0.21$ \\
$0.76 \pm 0.18$ \\
\end{tabular}

\begin{tabular}{c}
$\begin{array}{c}\text { Second quartile } \\
(n=6997)\end{array}$ \\
\hline $0.34 \pm 0.01$ \\
$(0.33-0.36)$ \\
$41.0 \pm 8.1$ \\
$1755(25.1)$ \\
$24.9 \pm 3.0$ \\
$84.6 \pm 8.9$ \\
$1305(31.1)$ \\
$736(10.5)$ \\
$117.3 \pm 14.3$ \\
$76.5 \pm 9.9$ \\
$5.7 \pm 0.4$ \\
$70.8 \pm 25.7$ \\
$5.6 \pm 0.3$ \\
$38 \pm 4$ \\
$2.57 \pm 0.97$ \\
$98.1 \pm 42.5$ \\
$0.13 \pm 0.29$ \\
$5.14 \pm 0.86$ \\
$1.69 \pm 0.84$ \\
$3.10 \pm 0.75$ \\
$3.08 \pm 0.72$ \\
$1.26 \pm 0.19$ \\
$3.87 \pm 0.84$ \\
$1.05 \pm 0.23$ \\
$1.42 \pm 0.22$ \\
$0.75 \pm 0.20$ \\
\hline
\end{tabular}

\begin{tabular}{c}
$\begin{array}{c}\text { Third quartile } \\
(n=6997)\end{array}$ \\
\hline $0.37 \pm 0.01$ \\
$(0.36-0.39)$ \\
$40.7 \pm 8.0$ \\
$2603(37.2)$ \\
$24.0 \pm 3.0$ \\
$82.3 \pm 9.0$ \\
$1067(25.4)$ \\
$662(9.5)$ \\
$115.2 \pm 14.2$ \\
$74.9 \pm 9.8$ \\
$5.6 \pm 0.4$ \\
$66.7 \pm 23.6$ \\
$5.6 \pm 0.3$ \\
$38 \pm 3$ \\
$2.40 \pm 0.90$ \\
$96.2 \pm 38.9$ \\
$0.12 \pm 0.32$ \\
$5.11 \pm 0.90$ \\
$1.37 \pm 0.67$ \\
$3.10 \pm 0.76$ \\
$3.05 \pm 0.76$ \\
$1.39 \pm 0.22$ \\
$3.72 \pm 0.78$ \\
$0.99 \pm 0.24$ \\
$1.44 \pm 0.22$ \\
$0.71 \pm 0.21$ \\
\hline
\end{tabular}

Fourth quartile $(n=6997)$ $0.43 \pm 0.05$ $(0.39-2.41)$ $39.8+7.9$ $4171(59.6)$

$22.6 \pm 2.9$

$77.2+9.3$

613 (15.2)

553 (7.9)

$112.1 \pm 14.2$

$72.4 \pm 9.7$

$5.5 \pm 0.5$

$61.1 \pm 20.1$

$5.6 \pm 0.3$

$38 \pm 3$

$2.15+0.77$

$96.2 \pm 43.5$

$0.12+0.44$

$5.07 \pm 0.88$

$1.00 \pm 0.58$

$2.95 \pm 0.78$

$2.84+0.78$

$1.66+0.31$

$3.41 \pm 0.88$

$0.89+0.23$

$1.50 \pm 0.24$

$0.61 \pm 0.21$

All $P<0.0001$ across all quartiles. HOMA, homeostasis model assessment. 
Table 2 Multivariate Cox proportional hazards regression analysis of HDL cholesterol and apolipoprotein A-I for the development of type 2 diabetes. Data are expressed as HR per 1 -s.D. increase in variables with $95 \% \mathrm{Cl}$.

\begin{tabular}{|c|c|c|c|c|}
\hline & \multicolumn{2}{|c|}{ HDL cholesterol } & \multicolumn{2}{|c|}{ Apolipoprotein A-I } \\
\hline & HR $(95 \% \mathrm{Cl})$ & $P$ & HR $(95 \% \mathrm{Cl})$ & $P$ \\
\hline Model I & $0.87(0.84-0.90)$ & $<0.001$ & $1.01(0.99-1.04)$ & 0.36 \\
\hline Model II & $0.91(0.88-0.95)$ & $<0.001$ & $1.03(1.00-1.06)$ & 0.06 \\
\hline Model III & $0.97(0.94-1.01)$ & 0.12 & 1.06 (1.03-1.09) & $<0.001$ \\
\hline Model IV & $1.00(0.96-1.05)$ & 0.91 & $1.10(1.06-1.14)$ & $<0.001$ \\
\hline Model V & $1.04(1.00-1.09)$ & 0.07 & $1.10(1.07-1.15)$ & $<0.001$ \\
\hline
\end{tabular}

Model I: no adjustment. Model II: adjusted for age and sex. Model III: model II + adjusted for BMI. Model IV: model III + adjusted for current smoking, systolic blood pressure, HbA1c, fasting serum insulin, and family history of diabetes. Model V: model IV + adjusted for triglyceride.

other HDL functions. Anti-inflammatory and antioxidative functions of HDLs have been described in addition to the innate immune function of HDLs as a defense against viral, bacterial, and parasitic infections (6). In addition, HDLs are directly involved in glucose metabolism through insulin secretion in pancreatic $\beta$-cells (13) and improvement of insulin sensitivity $(14,15)$. Although little is known about the role of apoA-I in the development of T2D, the findings of some recent studies are in agreement with the observations described in this study. Among 2111 middle-aged and elderly Turkish adults, subjects in the high serum apoA-I tertile had a nearly twofold increased risk for incident diabetes compared with subjects in the low serum apoA-I tertile during a 7.4 year follow-up. The authors of that study speculated that dysfunctional adiponectin associated with systemic inflammation and insulin resistance might transform apoA-I into proinflammatory particles and thus enhance the development of diabetes (9). Another study involving a Finnish cohort showed that apoA-I was an independent risk factor for the development of diabetes during a median 10.8-year follow-up (10).

Recent studies have suggested that the cardioprotective effects of HDLs can be altered in cases of systemic inflammation and T2D. In these situations, the lipid and protein composition of HDLs can be changed via oxidative processes. As a result, the protective effect of HDLs against CHD was lost and HDLs became proatherogenic, which are known as dysfunctional HDLs $(16,17,18)$. Ansell et al. (19) showed that HDLs from subjects with CHD were proinflammatory despite very high HDL-C levels, indicating that HDLs from CHD subjects are dysfunctional. In addition, treatment with torcetrapib, an inhibitor of cholesteryl ester transfer protein, was associated with an increase in cardiovascular events, despite a $72 \%$ increase in HDL-C levels (20). One possible mechanism for dysfunctional HDLs is the alteration in the protein composition of HDLs in the setting of systemic inflammation and T2D. In this situation, inflammatory cytokines such as plasma interleukin-6 levels are elevated and the liver consequently produces serum amyloid $\mathrm{A}$, which replaces apoA-I and paraoxonase 1 in HDL particles (16). In addition, myeloperoxidase specifically binds to apoA-I and produces reactive oxidative species. As a result, modified HDLs show an impaired cholesterol efflux in macrophages (17).

Human HDLs are a heterogeneous group of particles showing a range of densities, sizes, and electrophoretic mobilities as a result of the differences in the apolipoprotein compositions and lipid contents of HDL particles. It has been reported that the biological activities of HDLs differ according to HDL subpopulations (21). For example, the Framingham Offspring Study showed that large $\alpha 1$ HDL particles are inversely associated with CHD prevalence after adjusting for established CHD risk factors (22). Similarly, small discoid HDL particles are often elevated in patients with CHD, whereas large $\alpha 2$ HDL particles are decreased in patients with CHD (23). Previous studies suggested that the HA ratio reflects the core to surface ratio, or the particle size of HDL particles $(24,25,26)$. The HA ratio is lower in patients with metabolic syndrome

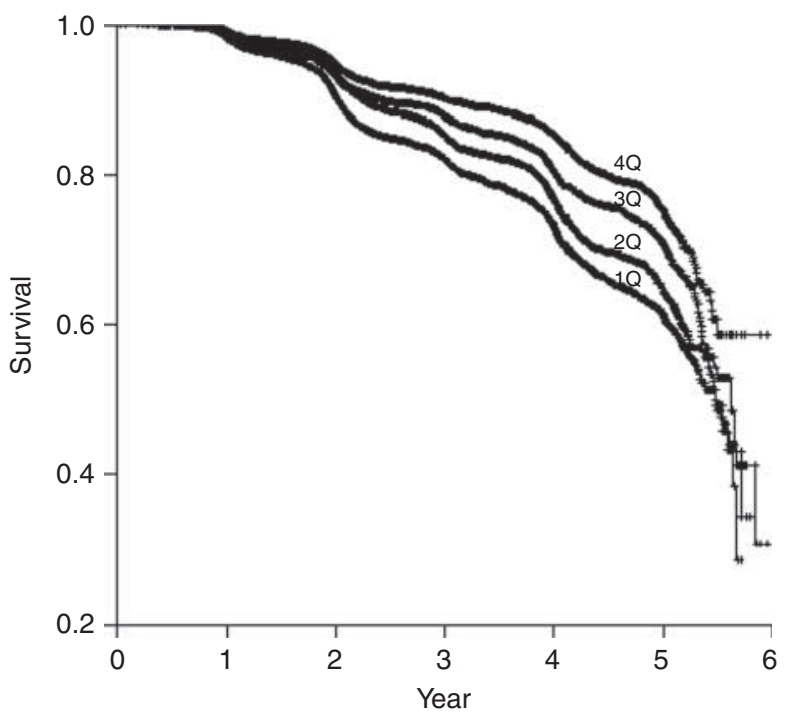

\section{Figure 1}

Kaplan-Meier survival curve for incident type 2 diabetes according to HDL cholesterol to apolipoprotein A-I ratio quartiles ( $P<0.0001$ across the quartiles). 
Table 3 Multivariate Cox proportional hazards regression analysis for the development of type 2 diabetes according to quartiles based on the ratio of HDL cholesterol to apolipoprotein A-I. Data are expressed as HR with $95 \% \mathrm{Cl}$.

\begin{tabular}{|c|c|c|c|c|}
\hline & $\begin{array}{c}\text { First } \\
\text { quartile }\end{array}$ & $\begin{array}{l}\text { Second } \\
\text { quartile }\end{array}$ & $\begin{array}{c}\text { Third } \\
\text { quartile }\end{array}$ & $\begin{array}{c}\text { Fourth } \\
\text { quartile }\end{array}$ \\
\hline Model I & 1 & $\begin{array}{c}0.85 \\
(0.78-0.91)\end{array}$ & $\begin{array}{c}0.69 \\
(0.64-0.75)\end{array}$ & $\begin{array}{c}0.55 \\
(0.50-0.60)\end{array}$ \\
\hline Model II & 1 & $\begin{array}{c}0.86 \\
(0.80-0.93)\end{array}$ & $\begin{array}{c}0.73 \\
(0.68-0.80)\end{array}$ & $\begin{array}{c}0.62 \\
(0.57-0.68)\end{array}$ \\
\hline Model III & 1 & $\begin{array}{c}0.90 \\
(0.83-0.97)\end{array}$ & $\begin{array}{c}0.79 \\
(0.73-0.86)\end{array}$ & $\begin{array}{c}0.72 \\
(0.66-0.79)\end{array}$ \\
\hline Model IV & 1 & $\begin{array}{c}0.88 \\
(0.80-0.97)\end{array}$ & $\begin{array}{c}0.76 \\
(0.69-0.84)\end{array}$ & $\begin{array}{c}0.69 \\
(0.61-0.78)\end{array}$ \\
\hline Model V & 1 & $\begin{array}{c}0.93 \\
(0.85-1.03)\end{array}$ & $\begin{array}{c}0.83 \\
(0.74-0.92)\end{array}$ & $\begin{array}{c}0.76 \\
(0.67-0.86)\end{array}$ \\
\hline
\end{tabular}

Reference quartile was defined as the first HDL cholesterol to apolipoprotein A-I ratio quartile. All $P<0.0001$ across all quartiles. Model I: no adjustment. Model II: adjusted for age and sex. Model III: model II+ adjusted for BMI. Model IV: model III+adjusted for current smoking, systolic blood pressure, $\mathrm{HbA} 1 \mathrm{c}$, fasting serum insulin, and family history of diabetes. Model V: model IV + adjusted for triglyceride.

compared with patients without metabolic syndrome, which is indicative of the presence of smaller denser HDL particles in patients with metabolic syndrome (26).

This study divided subjects by quartile according to the $\mathrm{HA}$ ratio. At baseline, as the $\mathrm{HA}$ ratio increased, BMI, waist circumference, blood pressure, fasting glucose, HOMA-IR, and hs-CRP levels decreased, which suggests that large HDL particle size is associated with healthy metabolic profiles. In addition, a higher HA ratio was associated with a lower incidence of T2D independent of other risk factors for T2D and serum triglyceride levels. Therefore, not only do large HDL particles have an effect on the development of CHD, but they also appear to be associated with a lower risk of developing T2D in subjects with IFG.

The results of this study may have clinical implications. The measurement of HDL-C alone is not sufficient to assess the association between cardiovascular risk and future development of T2D. At a given serum HDL-C concentration, additional measurement of serum apoA-I concentration may be needed to determine the relationship between cardiovascular risk and future development of T2D, that is, lower apoA-I at a given HDL-C concentration is associated with a lower cardiovascular risk and lower incidence of T2D, and thus, HA ratio may be used as a simple measurement for assessing cardiovascular risk and future development of T2D.

There are other limitations that should be considered in addition to the retrospective nature of this study. First, study subjects were in a state of IFG and thus might have some degree of insulin resistance compared with subjects with normal glucose tolerance. Therefore, it is possible that they already had some dysfunctional HDL particles and thus the results are less generalized. Secondly, the study had a relatively short follow-up period (2.81 years), which limited the interpretation of results in the setting of long-term effects of serum HDL-C and/or apoA-I on the development of T2D. In addition, subjects were selected from a population who visited the Health Screening Center for routine medical examinations, and thus, they were not representative of the general population and might be healthier than the general population.

In summary, the data suggest that the measurement of serum HDL-C alone is not sufficient to assess cardiometabolic risk, especially T2D. Simultaneous measurement of apoA-I provides a more accurate risk assessment for future development of T2D. Collectively, high HDL-C combined with low apoA-I was associated with a low incidence of T2D; however, the protective effect of HDL-C lessened as apoA-I increased, that is, a high HA ratio was associated with a lower risk of developing future T2D and could be used to assess the risk of T2D.

\section{Declaration of interest}

The authors declare that there is no conflict of interest that could be perceived as prejudicing the impartiality of the research reported.

\section{Funding}

This research did not receive any specific grant from any funding agency in the public, commercial or not-for-profit sector.

\section{References}

1 Castelli WP, Garrison RJ, Wilson PW, Abbott RD, Kalousdian S \& Kannel WB. Incidence of coronary heart disease and lipoprotein cholesterol levels. The Framingham Study. Journal of the American Medical Association 1986256 2835-2838. (doi:10.1001/jama.1986. $03380200073024)$

2 Turner RC, Millns H, Neil HA, Stratton IM, Manley SE, Matthews DR \& Holman RR. Risk factors for coronary artery disease in non-insulin dependent diabetes mellitus: United Kingdom Prospective Diabetes Study (UKPDS: 23). BMJ 1998316 823-828. (doi:10.1136/bmj.316. 7134.823)

3 Gordon DJ, Probstfield JL, Garrison RJ, Neaton JD, Castelli WP, Knoke JD, Jacobs DR Jr, Bangdiwala S \& Tyroler HA. High-density lipoprotein cholesterol and cardiovascular disease. Four prospective American studies. Circulation 198979 8-15. (doi:10.1161/ 01.CIR.79.1.8)

4 Lewis GF \& Rader DJ. New insights into the regulation of HDL metabolism and reverse cholesterol transport. Circulation Research 2005 96 1221-1232. (doi:10.1161/01.RES.0000170946.56981.5c) 
5 Leroux G, Lemieux I, Lamarche B, Cantin B, Dagenais GR, Lupien PJ \& Despres JP. Influence of triglyceride concentration on the relationship between lipoprotein cholesterol and apolipoprotein B and A-I levels. Metabolism 200049 53-61. (doi:10.1016/S0026-0495(00)90688-7)

6 Gordon SM, Hofmann S, Askew DS \& Davidson WS. High density lipoprotein: it's not just about lipid transport anymore. Trends in Endocrinology and Metabolism 201122 9-15. (doi:10.1016/j.tem.2010. 10.001)

7 von Eckardstein A, Schulte H \& Assmann G. Risk for diabetes mellitus in middle-aged Caucasian male participants of the PROCAM study: implications for the definition of impaired fasting glucose by the American Diabetes Association. Prospective Cardiovascular Munster. Journal of Clinical Endocrinology and Metabolism 200085 3101-3108. (doi:10.1210/jcem.85.9.6773)

8 Hwang YC, Ahn HY, Kim WJ, Park CY \& Park SW. Increased apoB/A-I ratio independently associated with type 2 diabetes mellitus: crosssectional study in a Korean population. Diabetic Medicine 201229 1165-1170. (doi:10.1111/j.1464-5491.2012.03622.x)

9 Onat A, Hergenc G, Bulur S, Ugur M, Kucukdurmaz Z \& Can G. The paradox of high apolipoprotein A-I levels independently predicting incident type- 2 diabetes among Turks. International Journal of Cardiology 2010142 72-79. (doi:10.1016/j.ijcard.2008.12.066)

10 Salomaa V, Havulinna A, Saarela O, Zeller T, Jousilahti P, Jula A, Muenzel T, Aromaa A, Evans A, Kuulasmaa K et al. Thirty-one novel biomarkers as predictors for clinically incident diabetes. PLOS ONE 2010 5 e10100. (doi:10.1371/journal.pone.0010100)

11 Matthews DR, Hosker JP, Rudenski AS, Naylor BA, Treacher DF \& Turner RC. Homeostasis model assessment: insulin resistance and beta-cell function from fasting plasma glucose and insulin concentrations in man. Diabetologia 198528 412-419. (doi:10.1007/ BF00280883)

12 American Diabetes Association. Diagnosis and classification of diabetes mellitus. Diabetes Care 201033 (Suppl 1) S62-S69. (doi:10.2337/ dc10-S062)

13 Drew BG, Duffy SJ, Formosa MF, Natoli AK, Henstridge DC, Penfold SA, Thomas WG, Mukhamedova N, de Courten B, Forbes JM et al. Highdensity lipoprotein modulates glucose metabolism in patients with type 2 diabetes mellitus. Circulation 2009119 2103-2111. (doi:10.1161/ CIRCULATIONAHA.108.843219)

14 Ruan X, Li Z, Zhang Y, Yang L, Pan Y, Wang Z, Feng GS \& Chen Y. Apolipoprotein A-I possesses an anti-obesity effect associated with increase of energy expenditure and up-regulation of UCP1 in brown fat. Journal of Cellular and Molecular Medicine 201115 763-772. (doi:10.1111/j.1582-4934.2010.01045.x)

15 Han R, Lai R, Ding Q, Wang Z, Luo X, Zhang Y, Cui G, He J, Liu W \& Chen Y. Apolipoprotein A-I stimulates AMP-activated protein kinase and improves glucose metabolism. Diabetologia 200750 1960-1968. (doi:10.1007/s00125-007-0752-7)
16 Kontush A \& Chapman MJ. Functionally defective high-density lipoprotein: a new therapeutic target at the crossroads of dyslipidemia, inflammation, and atherosclerosis. Pharmacological Reviews 200658 342-374. (doi:10.1124/pr.58.3.1)

17 Smith JD. Dysfunctional HDL as a diagnostic and therapeutic target. Arteriosclerosis, Thrombosis, and Vascular Biology 201030 151-155. (doi:10.1161/ATVBAHA.108.179226)

18 Barter PJ. The causes and consequences of low levels of high density lipoproteins in patients with diabetes. Diabetes \& Metabolism Journal 201135 101-106. (doi:10.4093/dmj.2011.35.2.101)

19 Ansell BJ, Navab M, Hama S, Kamranpour N, Fonarow G, Hough G, Rahmani S, Mottahedeh R, Dave R, Reddy ST et al. Inflammatory/ antiinflammatory properties of high-density lipoprotein distinguish patients from control subjects better than high-density lipoprotein cholesterol levels and are favorably affected by simvastatin treatment. Circulation 2003108 2751-2756. (doi:10.1161/01.CIR.0000103624. 14436.4B)

20 Barter PJ, Caulfield M, Eriksson M, Grundy SM, Kastelein JJ, Komajda M, Lopez-Sendon J, Mosca L, Tardif JC, Waters DD et al. Effects of torcetrapib in patients at high risk for coronary events. New England Journal of Medicine 2007357 2109-2122. (doi:10.1056/ NEJMoa0706628)

21 Camont L, Chapman MJ \& Kontush A. Biological activities of HDL subpopulations and their relevance to cardiovascular disease. Trends in Molecular Medicine 201117 594-603. (doi:10.1016/j.molmed. 2011.05.013)

22 Asztalos BF, Cupples LA, Demissie S, Horvath KV, Cox CE, Batista MC \& Schaefer EJ. High-density lipoprotein subpopulation profile and coronary heart disease prevalence in male participants of the Framingham Offspring Study. Arteriosclerosis, Thrombosis, and Vascular Biology 200424 2181-2187. (doi:10.1161/01.ATV. 0000146325.93749.a8)

23 Asztalos BF \& Schaefer EJ. High-density lipoprotein subpopulations in pathologic conditions. American Journal of Cardiology 200391 12E-17E. (doi:10.1016/S0002-9149(02)03383-0)

24 Miwa K, Yoshida N, Nakagawa K \& Inoue H. High-density lipoprotein particles are large in patients with variant angina. Cardiovascular Research 199837 729-737. (doi:10.1016/S0008-6363(97)00286-1)

25 Iribarren C, Belcher JD, Jacobs DR Jr, Gross MD, Schreiner PJ \& Sidney S. Relationship of lipoproteins, apolipoproteins, triglycerides and lipid ratios to plasma total cholesterol in young adults: the CARDIA Study. Coronary Artery Risk Development in Young Adults. Journal of Cardiovascular Risk 19963 391-396. (doi:10.1097/00043798199608000-00010)

26 Garin MC, Kalix B, Morabia A \& James RW. Small, dense lipoprotein particles and reduced paraoxonase- 1 in patients with the metabolic syndrome. Journal of Clinical Endocrinology and Metabolism 200590 2264-2269. (doi:10.1210/jc.2004-1295)

Received 11 March 2014

Revised version received 16 April 2014

Accepted 23 April 2014 